\title{
Chapitre II - Les sources
}

Maurice Wolkowitsch

\section{CpenEdition}

\section{Journals}

Édition électronique

URL : https://journals.openedition.org/rhcf/1302

DOI : 10.4000/rhcf.1302

Éditeur

Rails \& histoire

Édition imprimée

Date de publication : 10 février 2004

Pagination : 41-65

ISBN : 0996-9403

ISSN : 0996-9403

Référence électronique

Maurice Wolkowitsch, « Chapitre II - Les sources », Revue d'histoire des chemins de fer [En ligne], 30 | 2004, mis en ligne le 29 août 2012, consulté le 22 avril 2022. URL : http://journals.openedition.org/ rhcf/1302 ; DOI : https://doi.org/10.4000/rhcf.1302 


\section{Chapitre II}

\section{Les sources}

Cême si l'État donne avis et autorisation, les initiatives et les décisions relèvent des départements. Le conseil général tient un rôle fondamental. Des personnes physiques, des entreprises, suite à la loi de modernisation de l'économie de 1867 élargissant le champ d'application des sociétés anonymes, sont sollicitées pour participer à la réalisation des projets ferroviaires. Les communes sont soucieuses d'émettre des avis, les particuliers aussi. Les sources auxquelles le chercheur doit se reporter sont donc diverses.

\section{Les ressources des archives départementales et communales}

\section{. Comptes rendus des débats des assemblées des collectivités territoriales}

Les comptes rendus des débats des conseils généraux livrent les données fondamentales; en ces temps sans écologistes, la passion pour le chemin de fer était généralisée, chaque élu le voulait dans son canton, sa commune. Les conseils généraux consacrent de nombreuses séances au problème des chemins de fer; ils élaborent des projets parfois sans suite.

\section{$>$ Les débats parisiens et les élus locaux}

À Paris, les ingénieurs des Ponts discutent du choix des écartements et de l'opportunité même de construire des lignes secondaires ${ }^{1}$. Ces débats paraissent théoriques aux provinciaux.

Les élus avaient choisi l'écartement de 1,44 m, tant qu'ils poursuivaient les chimères de la concurrence et de l'intégration aux grands réseaux ; ils optèrent massivement pour la voie métrique, lorsqu'ils eurent découvert qu'elle permettait de construire près du double de kilomètres avec un même investissement. Un nouveau débat parisien s'engage lorsque Decauville démontre la fiabilité de la voie de 0,60 $\mathrm{m}$ utilisée à l'Exposition universelle de 1889 ; le coût de construction serait inférieur d'un tiers à celui de la voie métrique ; le débat reste parisien ; le peu d'intérêt des élus se traduit dans les faits ; moins de $2 \%$ du réseau sont construits à cet écartement : des lignes balnéaires autour de Caen

1- G. Ribeill, «Les chemins de fer d'intérêt local à l'épreuve du régime de 1880 : de graves pathologies congénitales?», RHCF 24-25, p. 104-137. 
et de Royan, la liaison de Pithiviers à Toury et $51 \mathrm{~km}$ dans le Tarn. Dans ce département le débat ne porte sur l'écartement qu'en apparence. Deux conceptions s'affrontent sur le réseau à construire mais l'opposition ne porte pas sur le choix technique. L'essentiel est ailleurs ${ }^{2}$.

Les ingénieurs des Ponts s'affrontaient aussi sur le rôle des lignes secondaires : apportent-elles aux grandes compagnies un nombre de tonnes $/ \mathrm{km}$ et voyageurs $/ \mathrm{km}$ qui justifie leur construction? Roulage, diligence et patache ne pourraient-ils acheminer sur de faibles distances ces trafics à meilleur compte jusqu'aux gares du réseau principal ? Ces considérations étaient aussi éloignées des réalités locales que peut l'être un rapport d'énarque au xxi siècle. L'ignorance du trafic local traduit la méconnaissance du terrain. L'acheminement par voie ferrée a permis un abaissement des prix de transport favorisant la diffusion des amendements comme l'approvisionnement des usines en matières premières : par exemple le prix de transport du kaolin sur $40 \mathrm{~km}$ vers les porcelaineries de Limoges a baissé de $27 \%{ }^{3}$. Pour les élus locaux, la cause était entendue, la nécessité du chemin de fer s'imposait à eux.

\section{$>$ Les débats à l'échelle des départements et des villes}

Les débats changent de contenu avec le temps. La première période, la plus riche en questions, correspond aux projets et constructions. Les conseillers fixent un programme de lignes à établir avec des priorités, le nombre et l'emplacement des stations, la participation financière du département, notamment en cas de déficit d'exploitation, suivant un régime défini pour la durée de la concession, comme si l'environnement économique était immuable. La deuxième période est celle de l'exploitation; les débats reflètent les mécontentements des usagers : tarifs trop élevés, services trop rares, horaires non respectés avec des incidences sur les correspondances avec les grandes compagnies qui refusent de prévoir des délais d'attente, inconfort des matériels, accidents trop fréquents, négligence et arrogance des personnels. Ces plaintes toujours renouvelées se traduisent en vœux transmis aux concessionnaires par le maire ou le président du conseil général. La troisième période est celle du déclin. Le souci des élus est de ne pas compromettre les finances départementales ou municipales en répondant aux appels à l'aide des compagnies qui menacent de cesser

2- O. Joseph, «Le réseau des destins croisés », RHCF 24-25, p. 245-269.

3- Prix du transport sur $40 \mathrm{~km}$, par tonne : roulage directe de la carrière à l'usine $12 \mathrm{~F}$. Par chemin de fer: camionnage de la carrière à la gare $3 \mathrm{~F}$, de la gare de Limoges à l'usine $2 \mathrm{~F}$, parcours ferroviaire 3,68 F, total 8,68 F. Économie 3,32 F (A.D. de la Haute-Vienne G.S.6.1) 
l'exploitation. Lorsque les exigences deviennent trop fortes, les débats portent éventuellement sur le rachat de la concession et, à un moment donné, sur la suspension de l'exploitation.

\section{$>$ Les débats à l'échelle des communes rurales}

Les conseils municipaux des communes rurales concernées de près ou de loin par le tracé d'une ligne émettent souvent des vœux, certains trouvent un écho au conseil général (emplacement des stations), d'autres leur sont plus particuliers. Ils demandent la création d'une halte supplémentaire, à défaut d'une station, ainsi le nom de la commune figurerait au livret horaire du chemin de fer. Des communes réclament un tracé traversant leur territoire ; il est exceptionnel de trouver un vœu défavorable au passage d'une voie ferrée ; mais on demande davantage de passages à niveau, même non gardés, pour limiter les allongements de parcours imposés par la coupure de la ligne aux exploitants pour atteindre certaines parcelles. La mise en marche de trains à l'occasion des pèlerinages est souhaitée. L'adaptation des horaires aux besoins des paysans fréquentant les foires et marchés est instamment réclamée, la proposition est faite pour ces jours-là de remplacer les convois mixtes marchandises-voyageurs par des trains affectés les uns aux voyageurs, les autres aux marchandises. Les conseils appuient aussi les plaintes concernant l'insuffisance des indemnités d'expropriation ${ }^{4}$ ou les souhaits de règlements assurant la sécurité des usagers de la route, véhicules, piétons et bétail en cas de partage de la chaussée avec un TVM. Un arrêté du préfet du Cher en 1897 répond à ce souci : «Le mouvement devra même être arrêté complètement toutes les fois que l'approche du train, en effrayant les chevaux ou autres animaux pourrait être une cause de désordre ou occasionner des accidents. Un aide du mécanicien ou un agent du convoi devra se porter immédiatement à la tête des chevaux, les prendre à la bride s'il est nécessaire, et les guider jusqu'à ce que toute crainte de danger ait disparu ${ }^{5}$.»

4- Exemple d'indemnités d'expropriation sur la rive droite de l'estuaire de la Gironde en 1885 (F par ha) :

- demande de l'exproprié :

$750 \quad 4000 \quad 4000 \quad 1200$

- offre d'indemnisation :

$\begin{array}{llll}270 & 560 & 979 & 400\end{array}$

- allocation prononcée par le juge des expropriations: $\begin{array}{lllll}350 & 1080 & 1329 & 700\end{array}$

- \% allocation / montant de la demande :

$\begin{array}{llll}47 & 27 & 33 & 58\end{array}$

5- Archives départementales du Cher, S 1821-1822, Organisation des transports routiers 1897-1908. 


\section{. Les autres documents disponibles dans les archives locales}

Les archives départementales conservent les doubles de tous les documents administratifs transmis par les services parisiens pour avis ou pour exécution : avis du Conseil d'État, du Conseil des Ponts, décisions ministérielles au cours des procédures imposées pour la construction d'une ligne secondaire. Les archives départementales conservent aussi tous les documents administratifs élaborés dans le département; ces derniers ne figurent pas toujours dans les dossiers des archives nationales : cette observation souligne la nécessité des recherches dans les dépôts départementaux et communaux des archives.

Hors les documents administratifs, les dossiers des archives livrent des informations multiples sur les chemins de fer secondaires. On y trouve souvent des références aux prises de position des chambres d'agriculture et des chambres de commerce et d'industrie, appuyées sur des exemples concrets de ce qu'on peut attendre d'une nouvelle desserte ferroviaire ; ces organismes font une critique circonstanciée de la tarification, réclament des tarifs spéciaux et des mesures d'harmonisation entre grandes compagnies et réseaux secondaires.

La presse locale rapporte aussi bien les débats politiques que les prises de position des milieux économiques ; mais elle publie aussi des articles de fond, soit sur la question des chemins de fer, soit sur des problèmes locaux; elle ouvre ses colonnes à des notables provinciaux. Les défenseurs de la voie d'eau face à l'extension du réseau ferré y trouvent un moyen d'expression.

Un ton plus polémique se manifeste dans une multitude de pamphlets, défendant des tracés alternatifs ou des emplacements de stations. Ils expriment des intérêts opposés, on y découvre des perspectives de trafic gonflées, une description partisane des avantages et des inconvénients des choix à opérer. À condition de les lire avec un esprit critique, il y a beaucoup à en apprendre.

\section{Les monographies de lignes ou réseaux (annexe 1)}

Ces travaux sont fondés sur les sources signalées ci-dessus ; ils s'appuient souvent sur un appel méticuleux aux dossiers des archives départementales. Les auteurs privilégient certains thèmes : la formation des réseaux, l'étude des tracés avec les intérêts divergents qui les soutiennent, les prévisions de trafic, les évolutions de la société et de 
l'économie. Certains auteurs s'intéressent aussi aux hommes qui ont créé et dirigé les entreprises, à la situation financière de ces dernières, aux conditions de travail et aux salaires des personnels ${ }^{6}$.

Les revues et les annuaires (annexe 2)

Les revues consacrées aux chemins de fer sont nombreuses. La Revue d'histoire des chemins de fer a publié des articles et un numéro entier consacré aux chemins de fer secondaires. Les communications au colloque de Châteauroux comportent des bibliographies ; trois intervenants ont traité des problèmes des sources : A. de Dieuleveult, M. Bergeyre, A. Le Guyader ${ }^{7}$.

D'autres revues, telle la Vie du Rail, traitent de tous les types de chemins de fer, d'autres concentrent leur intérêt sur les réseaux urbains et sur l'histoire des CFIL et des TVM, sauf exception, disparus. Ces publications servent souvent de vitrines à des associations dont les membres sont des passionnés et des amoureux du rail. Chemins de fer régionaux et urbains mérite d'être citée en exemple ${ }^{8}$. Le lecteur trouve des articles de fond consacrés à l'histoire, sans oublier les matériels et de brèves mises au point.

Les liaisons pouvant exister entre les entreprises doivent être recherchées. La présence des mêmes hommes au sein des conseils d'administration est un indice à ne pas négliger. Les annuaires des sociétés anonymes par actions de diverses époques retiennent les plus grandes entreprises, ce qui exclut l'immense majorité des compagnies des chemins de fer secondaires'. En revanche, l' Annuaire Marchal, publié à partir de 1885, est une source précieuse d'informations ; il devient l'Annuaire

6- Nous citons à titre d'exemples cinq monographies, mais la liste en serait très longue. Celle de B. Moreau nous a incité à nous intéresser activement aux chemins de fer secondaires.

- B. Moreau, Le Tacot, petit train berrichon, 2 tomes, Châteauroux, 1988, 122 p. et Issoudun, 1989, $176 \mathrm{p}$.

- G. Blondeau, A toute vapeur. Histoire et tribulations du chemin de fer de Sedan à Bouillon, Mezière-Charleville, Terres ardennaises, 1989, 118 p.

- J. Churot, Le Petit Train jaune de Cerdagne, Breil-sur-Roya, Éd. du Cabri, 1989, 94 p.

- P. Durbain, Le Chemin de fer de l'Entre-Deux-Mer, Mairie de Gignac, CLEM, 1998, $122 \mathrm{p}$.

- F. Ollivier, Le Blayais, bibliothèque de la Saugue-Saint-Trojan, 1993, 140 p.

7- Ouvr. cité, p. 301-348.

8- Cette revue bimestrielle est publiée par la Fédération des amis des chemins de fer secondaires et l'Union des exploitants des chemins de fer touristiques et de musées.

9- Annuaire Desfossés, Annuaire Chaix, Annuaire de la Société des éditions économiques et financières. 
des CFIL et des Tramways (Annuaire général des transports fusionnés). Chaque année, il fournit, pour certaines entreprises de toute importance, le réseau exploité, la liste des membres du conseil d'administration et des responsables des services, il précise l'organisation financière (capital actions et emprunts obligataires), la répartition des bénéfices et le bilan. C'est une source très intéressante même si toutes les entreprises ne sont pas traitées tous les ans et si les données sont plus ou moins complètes suivant les entreprises.

\section{Les difficultés propres à la recherche sur les chemins de fer secondaires}

La construction et l'exploitation de lignes de 10 à $40 \mathrm{~km}$ paraissaient compatibles avec l'initiative individuelle d'où, au moins à l'origine, la multiplication du nombre des entreprises et des concessions. Ce nombre doit être chiffré à un moment donné en relevant les concessions en cours, sans oublier les mutations subies par les entreprises au cours de leur histoire. En 1911, on dénombre 270 concessions : 95 pour les TVM, 85 pour les TV, 72 pour les CFIL (loi de 1880), 18 pour les CFIL (loi de 1865). Cet effectif est déjà une difficulté pour la recherche.

Les inventaires de la série $S$ des archives départementales, lorsqu'ils existent, sont moins précis que ceux de la série $\mathrm{F}^{14}$ des Archives nationales. Les dossiers classés par ligne sont définis par les seuls terminus, plus rarement par un contenu. L'exemple d'un chercheur ayant relevé environ 300 cotes dans le Calvados illustre ce propos ${ }^{10}$ : si pour quelques lignes, comme Caen à Courseulles, le contenu des dossiers est précisé, pour les autres on ne dispose que de deux noms (Bayeux à Arromanches ou Caumont à Villers-Bocage). Si retracer l'histoire d'une ligne de sa naissance à sa mort est généralement aisé, faire la synthèse de l'histoire d'une entreprise ou le compte des financements privés dans le cadre d'un département est plus aléatoire. Le rayonnement fréquent d'une entreprise sur plusieurs départements entraine une dispersion des archives qui ne facilite pas la recherche : Sud-France, par exemple, exploite des lignes dans cinq départements: Alpes-Maritimes, Var, Bouches-du-Rhône, Isère, Côte-d’Or.

10- A. S. Blin, maîtrise de l'université de Caen, 1995, «Les Chemins de fer d'intérêt local dans le Calvados entre 1871 et 1914 ». Pour la ligne de Caen à Courseulles, le contenu des liasses est le suivant : horaires : 1 liasse ; expropriations : 3 liasses ; concessions et subventions : 2 liasses ; plans et enquêtes sur l'emplacement des stations : 1 liasse ; divers pour la période 1880-1890: 1 liasse. 
Les archives n'ont pas toujours été déposées, notamment à l'époque pionnière; des comptes rendus de conseils d'administration, des éléments sur l'origine des entreprises font défaut; certains documents ont été détruits ou dans le meilleur des cas dorment dans un grenier au milieu de papiers de famille. Le chercheur peut avoir d'heureuses surprises : des compagnies comme celle du Chemin de fer de l'Est de Lyon ont remis de nombreux dossiers aux archives du Rhône ; ce n'est pas un cas unique. La vigilance de cheminots chercheurs a sauvé de la destruction des archives aussi importantes que celles de la SGCFE (pour. la CGEA voir annexe 3).

Deux autres difficultés sont à signaler, l'une relève de la terminologie, l'autre de la présentation statistique.

Une ligne de chemin de fer, ou de tout autre mode de transport, est un itinéraire entre deux points, parcouru par des services réguliers ; le terme est sans ambiguité. Il est souhaitable de préciser si l'exploitation est directe, concédée ou affermée et d'indiquer le statut sous lequel la ligne a été créée, CFIL, TVM...

La notion de réseau correspond à un ensemble cohérent de lignes exploitées par une même compagnie : n'écrit-on pas indifféremment les grands réseaux ou les grandes compagnies, lorsque nous évoquons le PLM, le Nord ou le PO ? Cette conception ne peut être transférée sans précaution au monde des chemins de fer secondaires. Des compagnies exploitent une ou deux lignes, voire plusieurs réseaux, sans que règne une parfaite connexité entre les relations assurées; l'emploi du mot réseau, si on l'utilise, ne rend pas compte de la réalité et se substitue abusivement à ceux de compagnie ou d'entreprise.

Une source de confusion tient aussi à l'expression « réseau départemental » : on l'utilise tantôt pour évaluer le kilométrage total de toutes les lignes d'un département aux mains de diverses entreprises, tantôt pour désigner le réseau d'une entreprise jouissant d'un quasimonopole dans un département, deux réalités très différentes. Une puissante entreprise s'est constituée sous le nom de Compagnie des chemins de fer départementaux, présente dans plusieurs départements ; mais toutes les sociétés portant l'appellation de Compagnie des chemins de fer départementaux avec désignation du département concerné sont indépendantes de la précédente ; le cas se vérifie entre autres dans les Ardennes et dans la Haute-Vienne.

Deux cent soixante-dix entreprises fournissent au ministère des Travaux publics la matière à l'établissement des statistiques nationales; elles ne se plient sans doute pas toutes aux mêmes règles. Dans l'ensemble, pour les compagnies exploitant une ou quelques lignes et moins de $100 \mathrm{~km}$, les données sont fournies pour chaque ligne ; pour les compagnies 
à assise départementale exploitant des réseaux de 100 à $400 \mathrm{~km}$, les données sont fournies pour la totalité du réseau ; pour les plus grandes entreprises, les chiffres sont fournis tantôt ligne par ligne, tantôt pour chaque réseau exploité, tantôt pour la totalité du kilométrage en service.

Nous avons pris connaissance des conditions dans lesquelles ont évolué les chemins de fer secondaires au cours de leurs périodes de progrès et de déclin. Nous avons succinctement évoqué la diversité des sources dont nous disposions, sans nous cacher leurs limites. Nous pouvons examiner l'histoire complexe des entreprises si nombreuses et si diverses par leur nature. Les hommes qui les ont créées méritent aussi l'intérêt. Certains ont conservé une vision locale ; avec ténacité, ils ont réussi à franchir tous les obstacles et à construire les quelques kilomètres de lignes conduisant de leur bourg à une station d'un grand réseau ; ils en attendaient tant! D'autres avec leurs diplômes des grandes écoles ou leurs capitaux, l'un n'excluant pas l'autre, ont, eux aussi, vaincu les obstacles et réussi à créer des réseaux. Les plus heureux ont fondé des dynasties qui ont traversé le $\mathrm{xx}^{\mathrm{e}}$ siècle et, pour quelques-uns, leur nom n'a pas disparu du monde des transports en ce début du xxi siècle. 


\section{Annexe 1 - Les principales collections d'ouvrages consacrés aux chemins de fer secondaires}

\section{- Éditions de l'Ormet}

André Jacquot et Pierre Laederich (sous la dir. de), Encyclopédie générale des transports. Chemins de fer, Valignat, Editions de l'Ormet, dates diverses.

Cette encyclopédie rassemble autant de fascicules que de départements; elle comprend pour chacun cartes des réseaux (tramways compris), détail des ouvertures et fermetures de lignes, notices historiques illustrées (classement par code postal).

\section{- Éditions du Cabri}

Henri Domengie a publié 10 volumes :

Vol. 1 à 4 : Trains oubliés [lignes secondaires du réseau principal].

Vol. 5 : Le Temps des tramways.

Vol. 6 : Les Petits Trains de jadis, France Sud-Est.

Vol. 7 : Les Petits Trains de jadis, France Sud-Ouest.

Vol. 8 : Les Petits Trains de jadis, Ouest de la France (disponible chez l'éditeur).

Vol. 9 : (avec José Banaudo), Les Petits Trains de jadis, Nord de la France.

Vol. 10 : (avec José Banaudo), Les Petits Trains de jadis, Est de la France.

\section{- Éditions Cénomane}

Un ouvrage grand format, illustré, est disponible sur le réseau d'intérêt local des départements suivants :

- Les Petits Trains de la Sarthe.

- Les Petits Trains d'Ille-et-Vilaine (épuisé, en bibliothèque).

- Le Petit Anjou $\left(\mathrm{d}^{\circ}\right)$.

- La Vendée des petits trains $\left(\mathrm{d}^{\circ}\right)$.

- Les Petits Trains des Côtes-du-Nord (en réimpr. en 2004).

- Petits trains de Touraine.

- Petits trains à l'assaut du Jura (épuisé, en bibliothèque).

- Quand les petits trains faisaient la Manche ( $\left.\mathrm{d}^{\circ}\right)$.

- Le Petit Train Longtemps (Ile de la Réunion).

- Les Petits Trains du Morbihan et de Loire-Inferrieure (épuisé, en bibliothèque).

- Les Petits Trains et tramways hauts-normands.

- Petits Trains de Savoie - Haute-Savoie.

- Calvados pour les petits trains.

- Finistère en petits trains.

- Chers petits trains du Loir-et-Cher.

- A travers Mayenne et Orne en petits trains.

- Petits trains au fil de la Vienne. 


\section{Annexe 2}

A- Table des matières de la revue Chemins de fer régionaux et urbains Index géographique établi par M. De Marco, xxxxde la FACS-UNECTO.

(Voir aussi le site Internet de l'association : http://www.trains-fr.org)

\section{1-Table des matières à compter du $n^{\circ} 36(165 / 1)$ Réseaux secondaires - France}

\begin{tabular}{|c|c|c|c|}
\hline № dépt. & $\begin{array}{c}\text { Nom } \\
\text { départements }\end{array}$ & Sujet articles & $N^{\circ}$ revue \\
\hline 1 & Ain & & \\
\hline \multirow{2}{*}{2} & \multirow{2}{*}{ Aisne } & \multirow{2}{*}{ Saint-Quentin, service de la voie de $60 \mathrm{~cm}$} & 218 \\
\hline & & & 97 \\
\hline 3 & Allier & & \\
\hline 4 & $\begin{array}{l}\text { Alpes de Haute- } \\
\text { Provence }\end{array}$ & & \\
\hline 5 & Hautes Alpes & & \\
\hline \multirow{2}{*}{6} & \multirow{2}{*}{ Alpes Maritimes } & Trams des Alpes-Maritimes et Sud-France & $146-150$ \\
\hline & & Ouest Varois & $220-224$ \\
\hline 7 & Ardèche & Vivarais & 237 \\
\hline 8 & Ardennes & & \\
\hline 9 & Ariège & & \\
\hline 10 & Aube & & \\
\hline 11 & Aude & Tramw ay à vapeur de l'Aude ( $n^{\circ}$ spécial) & 171 \\
\hline 12 & Aveyron & & \\
\hline 13 & Bouches-du-Rhône & Chemin de fer des Salins de Giraud & 258 \\
\hline 14 & Calvados & Chemin de fer du Calvados & 179-180-181 \\
\hline 15 & Cantal & & \\
\hline 16 & Charente & & \\
\hline \multirow{2}{*}{17} & \multirow{2}{*}{ Charente-maritime } & Tramw ay de Royan & \multirow{2}{*}{139} \\
\hline & & Société économique en Oléron et Saint-Trojan & \\
\hline 18 & Cher & & \\
\hline \multirow{2}{*}{19} & \multirow{2}{*}{ Corrèze } & Tramw ay de la Corrèze & 67 \\
\hline & & Po Corrèze ( $n^{\circ}$ spécial) & 93 \\
\hline 20 & Corse & $\left(n^{\circ}\right.$ spécial $)$ & 270 \\
\hline \multirow{2}{*}{21} & \multirow{2}{*}{ Côte-d'Or } & $\begin{array}{l}\text { Chemins de fer départementaux de la Côte } \\
\text { d'Or ( } n^{\circ} \text { spécial) }\end{array}$ & 155 \\
\hline & & $\begin{array}{l}\text { Chemin de fer électrique à voie de } 60 \text { de } \\
\text { Marigny-le-Cahouet }\end{array}$ & 259 \\
\hline \multirow{2}{*}{22} & \multirow{2}{*}{ Côte-d'Armor } & Chemin de fer des Côtes-du-Nord & 131 \\
\hline & & CEuvre de Harel-de-la-Noë & 147 \\
\hline 23 & Creuse & $\begin{array}{l}\text { Aucun chemin de fer secondaire n'a été } \\
\text { construit dans ce département }\end{array}$ & \\
\hline \multirow{2}{*}{24} & \multirow{2}{*}{ Dordogne } & Chemin de fer d'intérêt local de la Dordogne & 257 \\
\hline & & Tramw ay de la poudrière de Bergerac & 127 \\
\hline 25 & Doubs & & \\
\hline
\end{tabular}




\begin{tabular}{|c|c|c|c|}
\hline $\mathrm{N}^{\circ}$ dépt. & $\begin{array}{c}\text { Nom } \\
\text { départements }\end{array}$ & Sujet articles & $\mathrm{N}^{\circ}$ revue \\
\hline 26 & Drôme & & \\
\hline 27 & Eure & $\begin{array}{l}\text { Chemin de fer de Cormeilles à Glos } \\
\text { Montfort }\end{array}$ & $83-84$ \\
\hline \multirow{2}{*}{28} & \multirow{2}{*}{ Eure-et-Loir } & Tramw ay d'Eure-et-Loir & 262 \\
\hline & & Les chemins de fer en Eure-et-Loir & 99 \\
\hline 29 & Finistère & Voies ferrées départementales du Finistère & $175-176$ \\
\hline \multirow{4}{*}{30} & \multirow{4}{*}{ Gard } & Chemin de fer de Camargue & 143 \\
\hline & & Archéologie ferroviaire dans les Cévennes & 149 \\
\hline & & Archéologie ferroviaire en Arles & 154 \\
\hline & & Grand routage du Puits Destival à Arles & 203 \\
\hline \multirow{3}{*}{31} & \multirow{3}{*}{ Haute-Garonne } & Luchon Superbagnères & 253 \\
\hline & & VFDM Castres Revel & 149 \\
\hline & & Tramw ay du Val-d'Aran & 276 \\
\hline 32 & Gers & & \\
\hline 33 & Gironde & Tramw ay du Cap-Ferret & 244 \\
\hline 34 & Hérault & & \\
\hline 35 & Ille-et-Vilaine & (nº spécial) & 110 \\
\hline 36 & Indre & & \\
\hline 37 & Indre-et-Loire & & $92-93-94-96$ \\
\hline \multirow{2}{*}{38} & \multirow{2}{*}{ Isère } & Tramw ay ouest du Dauphiné & $202-203$ \\
\hline & & La Mure (nº spécial) & 250 \\
\hline 39 & Jura & A travers le Jura & 133 \\
\hline 40 & Landes & Chemin de fer La Teste à Cazaux & 249 \\
\hline 41 & Loir-et-cher & Tramw ay du Loir-et-Cher & $116-110$ \\
\hline \multirow{2}{*}{42} & \multirow{2}{*}{ Loire } & Monnerdil Feurs Panissieres & \multirow{2}{*}{164} \\
\hline & & Monnerdil Lartigues Lostouet Ballybunien & \\
\hline \multirow{2}{*}{43} & \multirow{2}{*}{ Lozère } & $\begin{array}{c}80 \text { ans de traction sur les Chemins de fer } \\
\text { départementaux Vivarais-Lozère }\end{array}$ & 125 \\
\hline & & $\begin{array}{l}\text { espérance de renaissance Flérac-Sainte- } \\
\text { Cécile (1969) }\end{array}$ & 99 \\
\hline 44 & Loire-Atlantique & Châteaubriant Erbray & 157 \\
\hline \multirow{2}{*}{45} & \multirow{2}{*}{ Loiret } & Tramw ay Pithiviers-Toury & 134 \\
\hline & & Tramw ay du Loiret & 128 \\
\hline \multicolumn{4}{|l|}{46} \\
\hline \multicolumn{4}{|l|}{47} \\
\hline \multicolumn{4}{|l|}{48} \\
\hline \multicolumn{4}{|l|}{49} \\
\hline 50 & Manche & $\begin{array}{c}\text { Tramw ays normands et desserte du Mont- } \\
\text { Saint-Michel }\end{array}$ & 271 \\
\hline
\end{tabular}




\begin{tabular}{|c|c|c|c|}
\hline$N^{\circ}$ dépt. & $\begin{array}{c}\text { Nom } \\
\text { départem ents }\end{array}$ & Sujet articles & $\mathrm{N}^{\circ}$ revue \\
\hline 51 & Marne & & \\
\hline 52 & Haute-Marne & $\begin{array}{c}\text { Chemin de fer de Foulain - Nogent-en- } \\
\text { Bassigny }\end{array}$ & 82 \\
\hline 53 & Mayenne & & \\
\hline 54 & Meurthe-et-Moselle & & \\
\hline 55 & Meuse & Chemin d'intérêt local Haironville Triaucourt & 264 \\
\hline 56 & Morbihan & La Trinité-Étel & 127 \\
\hline \multirow[t]{2}{*}{57} & \multirow{2}{*}{ Moselle } & $\begin{array}{l}\text { Chemin de fer de Lutzelbourg -Drulingen } \\
(67)\end{array}$ & 226 \\
\hline & & Novéant Gerze - Thionville Mondorf & 70 \\
\hline 58 & Nièvre & $\begin{array}{l}\text { Économiques Cosne Saint-Amand-en- } \\
\text { Puisaye }\end{array}$ & 269 \\
\hline 59 & Nord & & \\
\hline 60 & Oise & Chemin de fer Méru-Labosse & 274 \\
\hline \multirow{3}{*}{61} & \multirow{3}{*}{ Orne } & Voies ferrées économiques de l'Orne & 212 \\
\hline & & Voies ferrées économiques de Halouze & 215 \\
\hline & & Chemin de fer de la Grande-Trappe & 235 \\
\hline \multirow{5}{*}{62} & \multirow{5}{*}{ Pas-de-Calais } & Tramw ay de Quend à Fort-Mahon & 151 \\
\hline & & Berck-Plage - Paris-Plage & $72-75$ \\
\hline & & Tramw ay de l'Artois & $77-78$ \\
\hline & & Train de Guines & 280 \\
\hline & & Houillères Nord - Pas-de-Calais & 185 \\
\hline 63 & Puy de Dôme & Riom-Volvic & 87 \\
\hline \multirow{4}{*}{64} & \multirow{4}{*}{ Pyrénées-Atlantiques } & Bayonne - Biarritz & 132 \\
\hline & & Chemin de fer Haute Sioule & 228 \\
\hline & & Voies ferrées départementales du Midi & 120 \\
\hline & & Réseau basque et la Rhune & \\
\hline \multirow[t]{2}{*}{65} & \multirow[t]{2}{*}{ Hautes Pyrénées } & $\begin{array}{c}\text { Voies ferrées des Pyrénées - Tramw ay } \\
\text { de Bigorre }\end{array}$ & 96 \\
\hline & & PCL Pierrefitte - Cauterêt & $250-251$ \\
\hline 66 & Pyrénées Orientales & $\mathrm{N}^{\circ}$ spécial Cerdagne & 170 \\
\hline 67 & Bas-Rhin & & \\
\hline 68 & Haut-Rhin & Tramw ay de Ribeauvillié & 240 \\
\hline 69 & Rhône & Chemin de fer du Rhône et Loire & 173-174 \\
\hline 70 & Haute-Saône & Chemin de fer vicinaux de la Haute-Saône & 287 \\
\hline 71 & Saône-et-Loire & Chemin de fer du Rhône et Loire & 194 \\
\hline \multirow{3}{*}{72} & \multirow{3}{*}{ Sarthe } & Tramw ay de la Sarthe & 91 \\
\hline & & Le Mans - Mamers par Les TS en 1930 & 193 \\
\hline & & De Mamers - Saint-Calais à La Trans rap & 232 \\
\hline
\end{tabular}




\begin{tabular}{|c|c|c|c|}
\hline № dépt. & $\begin{array}{c}\text { Nom } \\
\text { départements }\end{array}$ & Sujet articles & $N^{\circ}$ revue \\
\hline \multirow{2}{*}{73} & \multirow{2}{*}{ Savoie } & Numéro spécial Savoie & 103 \\
\hline & & Chemin de fer du Revard & 252 \\
\hline \multirow{4}{*}{74} & \multirow{4}{*}{ Haute-Savoie } & Chemin de fer Montenvers & 165 \\
\hline & & Tramw ay du Mont-Blanc & 192 \\
\hline & & Ligne de Chamonix & 141 \\
\hline & & Funiculaire Rives-Thonon & 236 \\
\hline \multirow{5}{*}{75} & \multirow{5}{*}{ Paris et Couronne } & Chemin de fer de grande banlieue & \begin{tabular}{|c}
$66-72-73-74-$ \\
78 \\
\end{tabular} \\
\hline & & Petite ceinture & 75 \\
\hline & & Réouverture de la grande ceinture ouest & 221 \\
\hline & & $\begin{array}{l}\text { Chemins de fer industriels en région } \\
\text { parisienne }\end{array}$ & 265 \\
\hline & & Paris - Saint-Germain : de la SNCF au RER & 114 \\
\hline 76 & Seine-Maritime & Aumale - Envermeu & 246 \\
\hline \multirow{3}{*}{77} & \multirow{3}{*}{ Seine-et-Marne } & Montereau-Château Landon & 208 \\
\hline & & Sens - Aigreville & 209 \\
\hline & & Melun - Barbizon & 267 \\
\hline \multicolumn{4}{|l|}{78} \\
\hline 79 & Deux-Sèvres & Numéro spécial & 163 \\
\hline \multirow{2}{*}{80} & \multirow{2}{*}{ Somme } & Voie ferrée ayant desservi Ault & 249 \\
\hline & & Chemin de fer Baie-de-Somme & 273 \\
\hline \multirow{2}{*}{81} & \multirow{2}{*}{ Tarn } & Tramw ay du Tarn & 113 \\
\hline & & Tramw ay du Tarn et de Réalmont & 190 \\
\hline \multicolumn{4}{|l|}{82} \\
\hline \multicolumn{4}{|l|}{83} \\
\hline 84 & Var & Voir ouest Varois $(06)$ & \\
\hline \multirow{2}{*}{85} & \multirow{2}{*}{ Vendée } & Chemin de fer de Vendée & \multirow{2}{*}{$166-167-168$} \\
\hline & & Chemin de fer en Vendée & \\
\hline 86 & Vienne & & \\
\hline 87 & Haute-Vienne & Chemin de fer de Haute-Vienne & 68-69-73-76 \\
\hline \multirow{4}{*}{88} & \multirow{4}{*}{ Vosges } & Vallée de Celles - Raon l'Étape & 231 \\
\hline & & Traversée des Vosges & $216-217$ \\
\hline & & Remiremon t - Gérardmer - Schlucht & \\
\hline & & Remiremont - Munster & \\
\hline 89 & Yonne & Sens - Égreville & 209 \\
\hline 90 & Belfort & Chemin de fer d'intérêt local de Belfort & 79 \\
\hline
\end{tabular}


2-Table des matières à compter du $n^{\circ} 67(1965 / 1)$ Tramways urbains -électriques - vapeur - air condionné

\begin{tabular}{|c|c|c|c|}
\hline № dépt. & $\begin{array}{c}\text { Nom } \\
\text { départements }\end{array}$ & Sujet articles & $N^{\circ}$ revue \\
\hline 1 & Ain & & \\
\hline \multirow{3}{*}{2} & \multirow{3}{*}{ Aisne } & Laon de la crémaillère au Poma & 230 \\
\hline & & Tramw ay de Saint-Quentin et voie de 60 & 97 \\
\hline & & Tramw ay de Tergnier à Saint-Gobain & 245 \\
\hline 3 & Allier & Tramw ay Vichy - Cusset & 126 \\
\hline 4 & $\begin{array}{l}\text { Alpes de Haute- } \\
\text { Provence }\end{array}$ & & \\
\hline 5 & Hautes-Alpes & & \\
\hline \multirow{3}{*}{6} & \multirow{3}{*}{ Alpes-Maritimes } & Tramw ay de Monaco & $220-224$ \\
\hline & & $\begin{array}{l}\text { Tramw ay de Nice et du Littoral en 1914- } \\
1918\end{array}$ & 88 \\
\hline & & Sud-France et Tramw ay Alpes-Maritimes & $146-150$ \\
\hline \multicolumn{4}{|l|}{7} \\
\hline \multicolumn{4}{|l|}{8} \\
\hline \multicolumn{4}{|l|}{9} \\
\hline \multicolumn{4}{|l|}{10} \\
\hline \multicolumn{4}{|l|}{11} \\
\hline 12 & Aveyron & Tramw ay de Rodez & 85 \\
\hline \multicolumn{4}{|l|}{13} \\
\hline 14 & Calvados & Un tramw ay électrique en 1883 & 180 \\
\hline \multicolumn{4}{|l|}{15} \\
\hline \multicolumn{4}{|l|}{16} \\
\hline \multicolumn{4}{|l|}{17} \\
\hline 18 & Cher & Tramw ay de Bourges & 75 \\
\hline \multicolumn{4}{|l|}{19} \\
\hline \multicolumn{4}{|l|}{20} \\
\hline 21 & Côte-d'Or & Tramw ay de Dijon & 142 \\
\hline \multicolumn{4}{|l|}{22} \\
\hline \multicolumn{4}{|l|}{23} \\
\hline \multicolumn{4}{|l|}{24} \\
\hline 25 & Doubs & $\begin{array}{c}\text { Tramw ay de Besançon - Funiculaire de } \\
\text { Bregille }\end{array}$ & $99-222$ \\
\hline \multicolumn{4}{|l|}{26} \\
\hline 27 & Eure & Tramw ay d'Ebeuf & 92 \\
\hline \multicolumn{4}{|l|}{28} \\
\hline 29 & Finistère & Tramw ay de Brest au Conquet & 184 \\
\hline
\end{tabular}




\begin{tabular}{|c|c|c|c|}
\hline № dépt. & $\begin{array}{c}\text { Nom } \\
\text { départements }\end{array}$ & Sujet articles & $\mathrm{N}^{\circ}$ revue \\
\hline \multicolumn{4}{|l|}{30} \\
\hline \multicolumn{4}{|l|}{31} \\
\hline \multicolumn{4}{|l|}{32} \\
\hline \multicolumn{4}{|l|}{33} \\
\hline \multirow{4}{*}{34} & \multirow{4}{*}{ Hérault } & Tramw ay de Montpellier & 229 \\
\hline & & $\begin{array}{c}\text { Tramw ay de Montpellier à chevaux } \\
(1880-1883)\end{array}$ & 191 \\
\hline & & Tramw ay de Béziers & 233 \\
\hline & & Tramw ay de Sète & $201-202$ \\
\hline 35 & lle-et-Vilaine & Tramw ay de Rennes & 110 \\
\hline \multicolumn{4}{|l|}{36} \\
\hline 37 & Indre-et-Loire & Tramw ay de Tours ( $n^{\circ}$ spécial) & 98 \\
\hline 38 & Isère & Nouveau Tramw ay de Grenoble & 201 \\
\hline \multicolumn{4}{|l|}{39} \\
\hline \multicolumn{4}{|l|}{40} \\
\hline 41 & Loir-et-Cher & Tramw ay de Blois & 118 \\
\hline 42 & Loire & & \\
\hline \multicolumn{4}{|l|}{43} \\
\hline 44 & Loire-Atlantique & Tramw ay de La Baule (vapeur) & 126 \\
\hline \multirow{2}{*}{45} & \multirow{2}{*}{ Loiret } & Tramw ay d'Orléans & 138 \\
\hline & & Tramw ay de Montargis & 88 \\
\hline \multicolumn{4}{|l|}{46} \\
\hline \multicolumn{4}{|l|}{47} \\
\hline \multicolumn{4}{|l|}{48} \\
\hline \multicolumn{4}{|l|}{49} \\
\hline \multicolumn{4}{|l|}{50} \\
\hline \multicolumn{4}{|l|}{51} \\
\hline 52 & Haute-Marne & Langres & 241 \\
\hline \multicolumn{4}{|l|}{53} \\
\hline \multicolumn{4}{|l|}{54} \\
\hline \multicolumn{4}{|l|}{55} \\
\hline \multicolumn{4}{|l|}{56} \\
\hline \multirow{4}{*}{57} & \multirow{4}{*}{ Moselle } & Transports en commun de Metz & 102 \\
\hline & & Tramw ay de Metz en 1940-1945 & 261 \\
\hline & & $\begin{array}{c}\text { Tramw ay de Thionville et de la Vallée de la } \\
\text { Fentsch }\end{array}$ & 263 \\
\hline & & Tramw ay de Saint-Avold & 175 \\
\hline 58 & & & \\
\hline
\end{tabular}




\begin{tabular}{|c|c|c|c|}
\hline$N^{\circ}$ dépt. & $\begin{array}{c}\text { Nom } \\
\text { départem ents }\end{array}$ & Sujet articles & $N^{\circ}$ revue \\
\hline \multirow[b]{2}{*}{59} & \multirow[b]{2}{*}{ Nord } & Tramw ay de Cassel & 242 \\
\hline & & $\begin{array}{c}\text { Les tramw ays de Lille-Roubaix-Tourcoing } \\
\text { et de l'ElRT }\end{array}$ & 70 \\
\hline \multicolumn{4}{|l|}{60} \\
\hline \multicolumn{4}{|l|}{61} \\
\hline 62 & Pas-de-Calais & Tramw ay de Dunkerque & 279 \\
\hline 63 & Puy-de-Dôme & $\begin{array}{l}\text { Tramw ay de Clermont-Ferrand et du } \\
\text { Puy-de-Dôme }\end{array}$ & $255-256$ \\
\hline 64 & Pyrénées-Atlantiques & Réseaux de Bayonne à Biarritz & 132 \\
\hline 65 & Hautes-Pyrénées & Tramw ay de Lourdes & 227 \\
\hline \multicolumn{4}{|l|}{66} \\
\hline \multicolumn{4}{|l|}{67} \\
\hline 68 & Haut-Rhin & Tramw ay de Mulhouse ( $\mathrm{n}^{\circ}$ spécial) & 162 \\
\hline \multirow{3}{*}{69} & \multirow{3}{*}{ Rhône } & Lyon - Vénissieux (ligne des Belles Mères) & $177-178$ \\
\hline & & $\begin{array}{l}\text { Tramw ay des Chapeaux (Viricelles- } \\
\text { Chazelles-Saint-Symphorien) }\end{array}$ & 172 \\
\hline & & Tramw ay d'Ecully & 121 \\
\hline \multicolumn{4}{|l|}{70} \\
\hline \multicolumn{4}{|l|}{71} \\
\hline 72 & Sarthe & Tramw ay du Mans & 206 \\
\hline \multicolumn{4}{|l|}{73} \\
\hline \multicolumn{4}{|l|}{74} \\
\hline \multirow{6}{*}{75} & \multirow{6}{*}{ Seine et Couronne } & Les dépôts des Tramw ays de Paris & 117 \\
\hline & & Tramw ays dans banlieue Sud-Est de Paris & 101 \\
\hline & & Tramw ay de Paris à Saint-Germain & $122-123$ \\
\hline & & Tramw ay d'Aubervilliers & 275 \\
\hline & & Tramw ay de Paris lors des grèves & 174 \\
\hline & & $\begin{array}{l}\text { Les tramw ays de Paris et les Expositions } \\
\text { parisiennes }\end{array}$ & 169 \\
\hline \multirow{5}{*}{76} & \multirow{5}{*}{ Seine-Maritime } & Tramw ay du Havre ( $\mathrm{n}^{\circ}$ spécial) & 105 \\
\hline & & $\begin{array}{c}\text { Dieppe Tramw ay à cheval - électrique et } \\
\text { trolleybus (1912) }\end{array}$ & 241 \\
\hline & & Le Havre nouveau funiculaire & 115 \\
\hline & & Le Tréport Tramw ay & 135 \\
\hline & & Rouen - Transports urbains & 71-72-73-74 \\
\hline 77 & Seine-et-Marne & Tramw ay de Melun & 215 \\
\hline 78 & Seine-et-Oise & Tramw ay de Versailles ( $n^{\circ}$ spécial) & 144 \\
\hline 79 & & & \\
\hline
\end{tabular}




\begin{tabular}{|c|c|c|c|}
\hline № dépt. & $\begin{array}{c}\text { Nom } \\
\text { départements }\end{array}$ & Sujet articles & $N^{\circ}$ revue \\
\hline 80 & Somme & Tramw ay d'Amiens & 247 \\
\hline 81 & & & \\
\hline 82 & & & \\
\hline 83 & & Tramw ay d'Avignon & 211 \\
\hline 84 & Vaucluse & Tramw ay des Sables-d'Olonne & 168 \\
\hline 85 & Vendée & & \\
\hline 86 & & & \\
\hline 87 & & & \\
\hline 88 & & & \\
\hline 89 & & Tramw ay de Belfort & \\
\hline 90 & Belfort & & \\
\hline
\end{tabular}

\section{3- Matériel ferroviaire - Installations}

\begin{tabular}{|c|c|}
\hline Sujet articles & $\mathrm{N}^{\circ}$ revue \\
\hline \multicolumn{2}{|l|}{ France - vapeur et généralités } \\
\hline Liste du matériel conservé des chemins de fer secondaires & $67-79$ \\
\hline Utilisation mixte voie étroite - voie normale & 87 \\
\hline Monorail Lartigue & 97 \\
\hline $\begin{array}{l}\text { Locomotives trois essieux couplés des chemins de fer } \\
\text { départementaux }\end{array}$ & 140 \\
\hline Caractéristiques particulières des voies de mines et carrières & 161 \\
\hline Traction Air comprimé & 174 \\
\hline Histoire et évolution du rail de roulement & 182 \\
\hline Histoire et évolution du rail de tramw ay & 189 \\
\hline Transport des w agons voie normale sur voies étroites & 192 \\
\hline Patrimoine ferroviaire de la FACS ( $\mathrm{n}^{\circ}$ spécial $25^{\circ}$ anniversaire) & 200 \\
\hline $\begin{array}{l}\text { Machines à vapeur du chemin de fer minier de Cornas } \\
\text { (Cévennes) }\end{array}$ & 196 \\
\hline Mécanismes vapeur & 219 \\
\hline Tramw ays et Automotrices système Purrey & 223 \\
\hline $\begin{array}{l}\text { Sécurité sur les voies uniques des tramw ays et réseaux } \\
\text { secondaires }\end{array}$ & 243 \\
\hline Réalisations ferroviaires d’Amédée Bollée & 248 \\
\hline 031T Baldw in en 1914-1918 & 254 \\
\hline 030t Pinguely type 107 & 259 \\
\hline 40 ans de secondaires - 40 ans de touristiques & 261 \\
\hline
\end{tabular}




\begin{tabular}{|c|c|}
\hline Sujet articles & No revue \\
\hline La Société des chemins de fer sur route & 261 \\
\hline 130 Cail des chemins de fer départementaux Charente & 264 \\
\hline Locomotives à air comprimé Renault & 267 \\
\hline Locomotives Tubize et Blanc-Misseron & $\begin{array}{r}268-272- \\
274-276\end{array}$ \\
\hline Tramw ays mécaniques non classiques & 283 \\
\hline \multicolumn{2}{|l|}{ France - Traction moteurs thermiques } \\
\hline Autorails à voie métrique sur pneumatiques & 75 \\
\hline Autorails Renault & $90-109$ \\
\hline Autorails rapides Billiard Abidjan-Niger & 102 \\
\hline Autorails Billiard & 108 \\
\hline Nouveaux autorails des chemins de fer de Provence & 111 \\
\hline Autorails De Dion Bouton & 199 \\
\hline 3 autorails Brissonneau et Lotz en Allemagne & 276 \\
\hline Locomotives à voie métrique Brissonneau et Lotz & 276 \\
\hline \multicolumn{2}{|l|}{ France - Traction électrique et chemin de fer métropolitain } \\
\hline Traction électrique sur voies navigables & 81 \\
\hline Chemins de fer régionaux modernes & 86 \\
\hline Matériel auxiliaire du Métro de Paris & 111 \\
\hline Traction électrique dans les mines & 135 \\
\hline Signalisation du Métro de Paris & 178 \\
\hline Fin de la traction électrique à la mine de la Grand'Courbe & 193 \\
\hline Traction par accumulateurs sur les secondaires & 211 \\
\hline Funiculaires & 238 \\
\hline \multicolumn{2}{|l|}{ France - Air comprimé } \\
\hline Tramw ay à air comprimé & 285 \\
\hline Traction à air comprimé dans les mines & 126 \\
\hline Tramw ay à air comprimé Vichy - Cusset & 126 \\
\hline \multicolumn{2}{|l|}{ Étranger - Traction vapeur et généralités } \\
\hline $\begin{array}{l}\text { Premières locomotives à vapeur sur les chemins de fer suisses } \\
\text { à crémaillère }\end{array}$ & 76 \\
\hline Locomotives de Bosnie-Herzegovine & 114 \\
\hline \multicolumn{2}{|l|}{ Étranger - Traction électrique et divers } \\
\hline Projet SLR (1977) & 148 \\
\hline Nouveau train panoramique du MOB & 157 \\
\hline Nouvelles automotrices des Rochers de Naye & 180 \\
\hline Nouveau matériel du MOB & 191 \\
\hline Le GLT (1988) & 208 \\
\hline Locotracteur Hercule (Diesel) & 263 \\
\hline
\end{tabular}




\section{4- Réseaux étrangers - Europe}

\begin{tabular}{|c|c|}
\hline Sujet articles & $N^{0}$ revue \\
\hline \multicolumn{2}{|l|}{ Allemagne - RFA et RDA } \\
\hline La FACS en RDA (1982) & 172 \\
\hline Exploitation de la ligne Radeburg Radebeul & 128 \\
\hline Fin des tramw ays de Berlin ouest & 89 \\
\hline Tramw ay de Brême & 90 \\
\hline Voies étroites en RDA & 126 \\
\hline \multicolumn{2}{|l|}{ Autriche } \\
\hline Le rail en Autriche en 1968 & 90 \\
\hline Vallée de Steyr & 95 \\
\hline Tramw ay de Graz & 109 \\
\hline Tramw ays et Voies secondaires en 1969 & 98 \\
\hline Aperçu sur les Tramw ays de Vienne & 118 \\
\hline De Florisdorf à Kagran & 140 \\
\hline Point sur les voies étroites en 1977 & 145 \\
\hline \multicolumn{2}{|l|}{ Belgique } \\
\hline Bruxelles et ses transports & 82 \\
\hline Tramw ay du Hainaut en 1990 & 218 \\
\hline 100 ans de la SNCV & 197 \\
\hline \multicolumn{2}{|l|}{ Bosnie-Herzegovine } \\
\hline Locomotives (voir matériel) $n^{\circ} 1 / 4$ & 108 \\
\hline \multicolumn{2}{|l|}{ Espagne } \\
\hline Voyage circulaire sur les secondaires en 1965 & 69 \\
\hline Périple espagnol & 73 \\
\hline Du Sud-ouest à Bilbao - Etoile métrique de Bilbao & 84 \\
\hline Vacances en Catalogne - Olot Gerone - San Feliu de Guixols & 85 \\
\hline Transports publics en Espagne et au Portugal & $87-88$ \\
\hline Chemin de fer Andorre Escatron & 92 \\
\hline Crémaillère de Nuria & 92 \\
\hline Réseaux secondaires de la côte méditerranéenne & 101 \\
\hline Chemin de fer Reus-Salou & 102 \\
\hline Valence (nouvelles de 1983) & 176 \\
\hline Barcelone - Métro & 186 \\
\hline Nouvelles des FEVE en 1988 & 188 \\
\hline Valence des Voies ferrées d'intérêt local au RER & 224 \\
\hline Valence nouveaux tramw ays & 250 \\
\hline Eusko Tren - Bilbao - San Sebastian et lignes affluentes & 277 \\
\hline Musée ferroviaire de Ponferrada & 282 \\
\hline
\end{tabular}




\begin{tabular}{|c|c|}
\hline Sujet articles & $N^{\circ}$ revue \\
\hline \multicolumn{2}{|l|}{ Grande-Bretagne } \\
\hline Inverness à Kyle & 136 \\
\hline Le Snow don, Ravenglass and Eskdale & 141 \\
\hline Pays de Galles & $145-149$ \\
\hline Devon & 147 \\
\hline lle de Man & 149 \\
\hline Métro de Glasgow & 121 \\
\hline Tramw ay à caniveau de Londres & $195-196$ \\
\hline Hythe - Romney and Dymchurch Railw ay & 238 \\
\hline lle de Wright & 158 \\
\hline \multicolumn{2}{|l|}{ Confédération helvétique } \\
\hline Tramw ay du canton d'Esch & 83 \\
\hline Walden burgerbahn & 89 \\
\hline LLB (Loèche) & 89 \\
\hline MOB & 90 \\
\hline Chemin de fer Bremgarten Dietikon & 94 \\
\hline ORBE Chavornay & 95 \\
\hline Sernftalbahn & 102 \\
\hline Cent ans du Rigi & 106 \\
\hline Porrentruy Bonfol - chemin de fer du Jura & 109 \\
\hline Lugano Ponte Tresa & 109 \\
\hline Meirigen Innertkirchen & 136 \\
\hline Golden Pullman Express & 138 \\
\hline Transports en commun de Neuchatel & 147 \\
\hline Dernier train de la ligne sommitale de La Furka (1981) & 167 \\
\hline Ligne de la Furka (2000) & 278 \\
\hline Chemin de fer Bière Apples-Morgues & 177 \\
\hline Chemin de fer Appenzel & 184 \\
\hline Nyon Saint-Cergue, modernisation & 187 \\
\hline Les chemins de fer du Jura ont 100 ans & 189 \\
\hline Chemin de fer du Rothorn & 207 \\
\hline Tramw ay de Sarrelouis & 204 \\
\hline \multicolumn{2}{|l|}{ Hollande } \\
\hline Tramw ay de Maastricht & 235 \\
\hline \multicolumn{2}{|l|}{ Italie } \\
\hline Chemin de fer de Trente à Male & 88 \\
\hline Chemin de fer de Calabre (Région de Barr) & 129 \\
\hline Chemin de fer de Sardaigne & 156 \\
\hline
\end{tabular}




\begin{tabular}{|c|c|}
\hline Sujet articles & $\mathrm{N}^{\circ}$ revue \\
\hline \multicolumn{2}{|l|}{ Grèce } \\
\hline Chemin de fer à voie étroite & 173 \\
\hline La FACS en Grèce & 175 \\
\hline Voie étroite et chemins de fer touristiques & 266 \\
\hline \multicolumn{2}{|l|}{ Hongrie } \\
\hline Crémaillère de Budapest & 128 \\
\hline Budapest - Les métros & 133 \\
\hline Lignes suburbaines de Budapest & 141 \\
\hline \multicolumn{2}{|l|}{ Lituanie } \\
\hline Voies étroites en Lituanie & 271 \\
\hline \multicolumn{2}{|l|}{ Luxembourg } \\
\hline Tramw ay de Luxembourg & 68 \\
\hline Chemin de fer à voie étroite du Luxembourg & $136-137$ \\
\hline Nouvelles du Luxembourg (2001) & 284 \\
\hline \multicolumn{2}{|l|}{ Pologne } \\
\hline Voie étroite en Pologne (1986) & 193 \\
\hline \multicolumn{2}{|l|}{ Portugal } \\
\hline Transports publics en Espagne et au Portugal & $87-88$ \\
\hline Tramw ay de Lisbonne & $197-198$ \\
\hline Situation des tramw ays au Portugal en 1994 & 247 \\
\hline Funiculaires de Braga et Viana do Castelo & 279 \\
\hline \multicolumn{2}{|l|}{ Roumanie } \\
\hline Lignes à voie étroite et crémaillère & 129 \\
\hline \multicolumn{2}{|l|}{ Suède } \\
\hline $\begin{array}{l}\text { Inversion du sens de circulation et son effet sur les transports } \\
\text { publics }\end{array}$ & 84 \\
\hline
\end{tabular}

\section{4- Réseaux non européens}

\begin{tabular}{|c|c|}
\hline Sujet articles & $N^{\circ}$ revue \\
\hline \multicolumn{2}{|l|}{ Afrique } \\
\hline Transports d'Alger & 210 \\
\hline Autorails dans les colonies françaises & 281 \\
\hline Voie de 60 en Afrique du Sud & 154 \\
\hline Les chemins de fer à voie métrique d'Algérie & 286 \\
\hline \multicolumn{2}{|l|}{ Amérique du Nord } \\
\hline Impressions sur les transports de New -York (1970) & 97 \\
\hline Impressions d’Amérique (1972) & 115 \\
\hline White Pass and Yukon Route & 119 \\
\hline
\end{tabular}




\begin{tabular}{|c|c|}
\hline Sujet articles & $N^{\circ}$ revue \\
\hline \multicolumn{2}{|l|}{ Amérique du Nord (suite) } \\
\hline Voies étroites dans le Colorado & 124-173 \\
\hline Elevated de New York & 124 \\
\hline Elevated de New York et le centenaire du pont de Brooklyn & 187 \\
\hline La grande époque des tramw ays aux USA & 124 \\
\hline Tramw ay moderne aux USA & 129 \\
\hline $\begin{array}{l}\text { Crémaillères de Mont Washington et Manitou and Pike's Peak } \\
\text { Railw ay }\end{array}$ & 161 \\
\hline Tramw ay de San Francisco (1984) & 173 \\
\hline Interurbains aux USA & 204 \\
\hline \multicolumn{2}{|l|}{ Amérique du Sud - Chili } \\
\hline Chemin de fer militaire de Puente Alta a el Volcan & 186 \\
\hline \multicolumn{2}{|l|}{ Asie } \\
\hline Chemin de fer du Hedjaz - Syrie & 281 \\
\hline Yunnan - Indochine & $\begin{array}{l}166-167- \\
239-284\end{array}$ \\
\hline Cambodge - situation en 1998 & 267 \\
\hline Sri Lanka - spécial vapeur 2'6" & 180 \\
\hline Chine - Tramw ay & 186 \\
\hline
\end{tabular}




\section{B- Table des matières de la revue Magazine des tramways à vapeur et des secondaires établie par Frédéric}

Delaitre (site Internet : www.fdelaitre.com)

La revue MTVS - Magazine des Tramways à vapeur et des Secondaires, " revue trimestrielle des Amis du rail secondaire et touristique », a été publiée de 1977 à 1989 par Jean-Claude Riffaud, directeur de la publication et auteur de la plupart des monographies, décédé en 1989 (rédacteur en chef : Jacques Renaud). La collection (52 livraisons de 56 pages en moyenne, format 21 x $29,5 \mathrm{~cm}$, illustrations) représente une somme documentaire indispensable pour le chercheur intéressé par l'histoire des chemins de fer secondaires.

\begin{tabular}{|c|c|c|c|}
\hline $\mathbf{N}^{\circ}$ & Millésime & Sujet & Auteur(s) \\
\hline 1 & $1977-1$ & Le chemin de fer de Valmondois à Marines. & J.-C. Riffaud \\
\hline 2 & $1977-2$ & $\begin{array}{l}\text { Le tramw ay de Villiers-le-Bel et compléments sur le chemin de } \\
\text { fer de Valmondois à Marines. }\end{array}$ & J.-C. Riffaud \\
\hline 3 & $1977-3$ & $\begin{array}{l}\text { La Société Centrale de Chemins de Fer et d'Entreprises et ses } \\
\text { automotrices }\end{array}$ & J.-C. Riffaud \\
\hline 4 & $1977-4$ & $\begin{array}{l}\text { Les autorails des Ateliers CFD de Montmirail - Les locomotives } \\
\text { type Sarthe - Les locomotives Mallet des CFD - Le chemin de } \\
\text { fer de Chinon à Richelieu. }\end{array}$ & J.-C. Riffaud \\
\hline 5 & $1978-1$ & Les chemins de fer de la Corse. & J.-C. Riffaud \\
\hline 6 & $1978-2$ & Les chemins de fer départementaux de la Mayenne. & J.-C. Riffaud \\
\hline 7 & $1978-3$ & Les locotracteurs CFD des Ateliers de Montmirail. & J.-C. Riffaud \\
\hline 8 & $1978-4$ & Le chemin du fer du Blanc à Argent (réédité en 1981 et 1984) & J.-C. Riffaud \\
\hline 9 & $1979-1$ & Les chemins de fer vicinaux de la Haute-Saône & J.-C. Riffaud \\
\hline 10 & $1979-2$ & Les chemins de fer vicinaux du Jura & J.-C. Riffaud \\
\hline 11 & $1979-3$ & La ligne de I'Yonne des Chemins de fer départementaux & J.-C. Riffaud \\
\hline 12 & $1979-4$ & La ligne de la Lozère des Chemins de fer départementaux & J.-C. Riffaud \\
\hline 13 & $1980-1$ & Le réseau du Vivarais des Chemins de fer départementaux & J.-C. Riffaud \\
\hline 14 & $1980-2$ & Les chemins de fer du Sud-Ouest & Henri Domengie \\
\hline 15 & $1980-3$ & $\begin{array}{l}\text { Le réseau des Charentes et des Deux-Sèvres des Chemins de } \\
\text { fer départementaux }\end{array}$ & J.-C. Riffaud \\
\hline 16 & $1980-4$ & $\begin{array}{l}\text { Le réseau de Seine-et-Marne des Chemins de fer } \\
\text { départementaux }\end{array}$ & J.-C. Riffaud \\
\hline 17 & $1981-1$ & $\begin{array}{l}\text { Le réseau de Saône-et-Loire des Chemins de fer } \\
\text { départementaux }\end{array}$ & J.-C. Riffaud \\
\hline 18 & $1981-2$ & $\begin{array}{l}\text { Les réseaux d'Indre-et-Loire des Chemins de fer } \\
\text { départementaux }\end{array}$ & J.-C. Riffaud \\
\hline 19 & $1981-3$ & $\begin{array}{l}\text { Les Chemins de fer départementaux (1881-1981) Numéro } \\
\text { Spécial Centenaire }\end{array}$ & J.-C. Riffaud \\
\hline
\end{tabular}




\begin{tabular}{|c|c|c|c|}
\hline $\mathbf{N}^{\circ}$ & Millésime & Sujet & Auteur(s) \\
\hline 20 & $1981-4$ & Les tramw ays de Saint-Etienne et des environs & Jean-Paul Rahon \\
\hline 21 & $1982-1$ & Les automotrices Tartary et de Dion-Bouton & J.-C. Riffaud \\
\hline 22 & $1982-3$ & Les tramw ays de l'Ardèche & Henri Domengie \\
\hline 23 & $1982-3$ & Les Chemins de fer départementaux de l'Aube & J.-C. Riffaud \\
\hline 24 & $1982-4$ & Les automotrices Billard & J.-C. Riffaud \\
\hline 25 & $1983-1$ & Le tramw ay de Lunéville à Einville & $\begin{array}{l}\text { Maurice Bourgeois } \\
\text { et Jacques Renaud }\end{array}$ \\
\hline 26 & $1983-2$ & Le chemin de fer Pau-Oloron-Mauléon & J.-C. Riffaud \\
\hline 27 & $1983-3$ & Le chemin de fer de Lunéville à Blamont et Badonviller & $\begin{array}{l}\text { Maurice Bourgeois } \\
\text { et Jacques Renaud }\end{array}$ \\
\hline 28 & $1983-4$ & Les automotrices Renault & J.-C. Riffaud \\
\hline 29 & $1984-1$ & Les tramw ays de la Sarthe & $\begin{array}{l}\text { J.-C. Riffaud et } \\
\text { Jacques Renaud }\end{array}$ \\
\hline 30 & $1984-2$ & Le chemin de fer de Nantes à Legé et à Rocheservière & J.-C. Riffaud \\
\hline 31 & $1984-3$ & Le chemin de fer de Valmondois à Marines & $\begin{array}{l}\text { J.-C. Riffaud et } \\
\text { Jacques Renaud }\end{array}$ \\
\hline 32 & 1984-4 & Les chemins de fer de la Corse (réédition) & J.-C.Riffaud \\
\hline 33 & $1985-1$ & Le tramw ay à vapeur de la Chalosse et du Béarn & J.-C. Riffaud \\
\hline 34 & $1985-2$ & Le Tramw ay de Bordeaux à Cadillac & $\begin{array}{l}\text { J.-C. Riffaud et } \\
\text { J. Renaud }\end{array}$ \\
\hline 35 & $1985-3$ & Le chemin de fer d'Orange à Buis-les-Baronnies & J.-C. Riffaud \\
\hline 36 & $1985-4$ & Les automotrices Crochat et Brissonneau-Lotz & J.-C. Riffaud \\
\hline 37 & $1986-1$ & Le réseau de Seine-et-Marne des Chemins de fer économiques & $\begin{array}{l}\text { J.-C. Riffaud et } \\
\text { J. Renaud }\end{array}$ \\
\hline 38 & $1986-2$ & Numéro spécial locotracteurs & J.-C. Riffaud \\
\hline 39 & $1986-3$ & Les chemins de fer départementaux de la Dordogne & $\begin{array}{l}\text { J.-C. Riffaud et } \\
\text { Bernard Jacquet }\end{array}$ \\
\hline 40 & $1986-4$ & 1976-1986 Dix ans d'évolution sur les métriques français & J.-C. Riffaud \\
\hline 41 & $1987-1$ & Les chemins de fer départementaux de la Haute-Vienne & J.-C. Riffaud \\
\hline 42 & $1987-2$ & Les chemins de fer du Doubs (Première partie) & J.-C. Riffaud \\
\hline 43 & $1987-3$ & Les chemins de fer du Doubs (Deuxième partie) & J.-C. Riffaud \\
\hline \multirow{2}{*}{44} & \multirow{2}{*}{$1987-4$} & Le chemin de fer de Lagny à Mortcerf & \multirow{2}{*}{ J.-C. Riffaud } \\
\hline & & Les tracteurs CFD de 1977 à 1987 & \\
\hline 45 & $1988-1$ & Le tramw ay de Meaux à Dammartin & J.-C. Riffaud \\
\hline 46 & $1988-2$ & Les tramw ays des Alpes-Maritimes & Raymond Delaveau \\
\hline 47 & $1988-3$ & Les tramw ays du Lot-et-Garonne & Henri Domengie \\
\hline 48 & $1988-4$ & Les locomotives des CFD (1re partie) & J.C Riffaud \\
\hline 49 & $1989-1$ & Les locomotives des CFD (2e partie) Les locotracteurs & J.-C. Riffaud \\
\hline 50 & $1989-2$ & Répertoire général des voies ferrées d'intérêt local en 1927 & - \\
\hline \multirow{2}{*}{51} & \multirow{2}{*}{$1989-3$} & Répertoire général des voies ferrées d'intérêt général & \\
\hline & & Les automotrices des réseaux secondaires & \\
\hline 52 & $1989-4$ & Les chemins de fer du Beaujolais & Henri Domengie \\
\hline
\end{tabular}




\section{Annexe 3 - Les archives d'entreprises}

Les archives des compagnies de chemins de fer secondaires sont rares. Des fonds peuvent se trouver dans les archives départementales. De leur côté, les Archives nationales conservent un dépôt de la Compagnie générale d'entreprises automobiles - CGEA qui réunit un certain nombre d'entreprises de transports et qu'il est utile de signaler (à l'exception de la SGCFE). Les fonds intéressant les CFIL et tramways sont:

- Chemins de fer d'intérêt local du nord de la France ;

- Chemins de fer du Calvados ;

- Chemins de fer du Güé à Menaucourt ;

- Chemins de fer et tramways du Var et du Gard;

- Chemins de fer secondaires du Nord-Est;

- Chemins de fer secondaires et transport automobile ;

- Compagnie algérienne de transport en commun ;

- Compagnie des tramways de Rouen;

- Compagnie de tramways de Bourges ;

- Compagnie de tramways de Cambrai et de Saint-Quentin ;

- Compagnie de tramways de Poitiers ;

- Compagnie française des tramways électriques et omnibus de Bordeaux ; Compagnie française de transport automobile (CFTA);

- Compagnie générale des tramways du Havre ;

- Compagnie générale de transport professionnel ;

- Compagnie générale française de tramways;

- Compagnie générale française de tramways. Comité central d'entreprise de la Compagnie générale parisienne de tramways ;

- Compagnie tunisienne d'électricité et de transport ;

- Coopérative de consommation du personnel de la Compagnie générale d'entreprises automobiles;

- Omnium de transports et de nettoiement ;

- Régie mixte des transports toulonnais ;

- Société des tramways d'Amiens;

- Société des transports en commun d'Hanoi;

- Société versaillaise d'exploitation.

Dates extrêmes : 1921-1994

Importance matérielle : 270 unités documentaires

Modalités d'entrée : dépôt de la Compagnie générale d'entreprises automobiles

Conditions d'accès : communicable suivant les délais indiqués dans le contrat Instrument de recherche : répertoire méthodique CAMT en ligne à l'adresse : http://www.archivesnationales.culture.gouv.fr/camt/fr/inventaires1996/1996027.html 
